\title{
AUTOMORPHISM GROUPS OF SOME AFFINE AND FINITE TYPE ARTIN GROUPS
}

\author{
Ruth Charney and John Crisp
}

\begin{abstract}
We observe that, for fixed $n \geq 3$, each of the Artin groups of finite type $A_{n}, B_{n}=C_{n}$, and affine type $\tilde{A}_{n-1}$ and $\tilde{C}_{n-1}$ is a central extension of a finite index subgroup of the mapping class group of the $(n+2)$-punctured sphere. (The centre is trivial in the affine case and infinite cyclic in the finite type cases). Using results of Ivanov and Korkmaz on abstract commensurators of surface mapping class groups we are able to determine the automorphism groups of each member of these four infinite families of Artin groups.
\end{abstract}

A rank $n$ Coxeter matrix is a symmetric $n \times n$ matrix $M$ with integer entries $m_{i j} \in \mathbb{N} \cup\{\infty\}$ where $m_{i j} \geq 2$ for $i \neq j$, and $m_{i i}=1$ for all $1 \leq i \leq n$. Given any rank $n$ Coxeter matrix $M$, the Artin group of type $M$ is defined by the presentation

$$
A(M) \cong\left\langle s_{1}, \ldots, s_{n}\right| \underbrace{s_{i} s_{j} s_{i} \ldots}_{m_{i j}}=\underbrace{s_{j} s_{i} s_{j} \ldots}_{m_{i j}} \text { for all } i \neq j, m_{i j} \neq \infty\rangle .
$$

Adding the relations $s_{i}^{2}=1$ to this presentation yields a presentation of the Coxeter group of type $M$ generated by standard reflections $s_{i}$ and such that the rotation $s_{i} s_{j}$ has order $m_{i j}$, for all $1 \leq i, j \leq n$. A Coxeter matrix $M$ and its Artin group $A(M)$ are said to be of finite type if the associated Coxeter group $W(M)$ is finite, and of affine (or Euclidean) type if $W(M)$ acts as a proper, cocompact group of isometries on some Euclidean space with the generators $s_{1}, \ldots, s_{n}$ acting as affine reflections.

The information contained in the Coxeter matrix $M$ is often displayed in the form of a graph, the Coxeter graph, whose vertices are numbered $1, \ldots, n$ and which has an edge labelled $m_{i j}$ between the vertices $i$ and $j$ whenever $m_{i j} \geq 3$ or $m_{i j}=\infty$. With this particular convention, one usually suppresses the labels which are equal to 3 (but not the corresponding edges!). Note that the absence of an edge between two vertices indicates that the corresponding generators of $A(M)$ commute. We say that an Artin group is irreducible if its Coxeter graph is connected and observe that every Artin group is isomorphic to a direct product of irreducible Artin groups corresponding to the connected components of its Coxeter graph.

In this paper we concern ourselves with the following four infinite families of Artin groups $A=A(M)$ (see Figure 1): the irreducible finite types $M=A_{n}$,

Received August 26, 2004.

Charney was partially supported by NSF grant DMS-0104026. 
$B_{n}$ and irreducible affine types $\tilde{A}_{n-1}$ and $\tilde{C}_{n-1}$, of rank $n \geq 3$ in each case. (We refer to [3] for the complete classification of irreducible finite and affine Coxeter systems.) For each Artin group $A$ on this list, we determine its automorphism group $\operatorname{Aut}(A)$, its outer automorphism group $\operatorname{Out}(A)=\operatorname{Aut}(A) / \operatorname{Inn}(A)$, and the abstract commensurator group $\operatorname{Comm}(A / Z)$ of the group modulo its centre (see Definition 3).
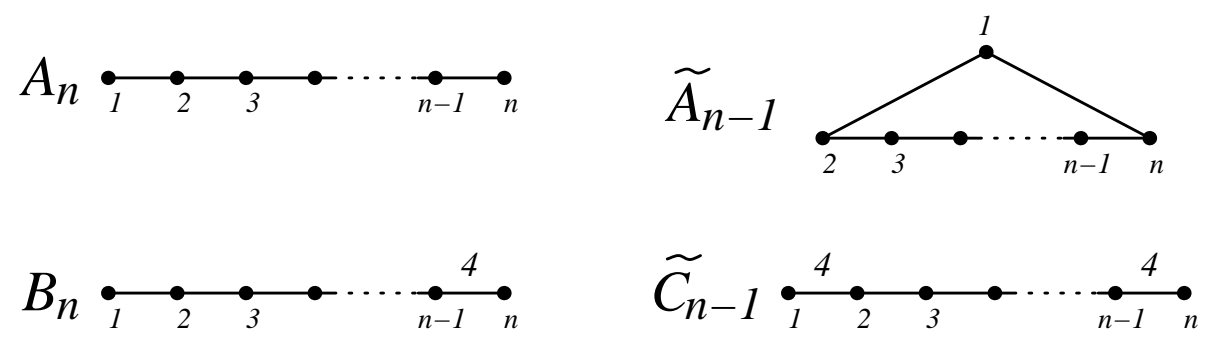

Figure 1. Coxeter graphs of finite type $A_{n}, B_{n}$ and affine type $\tilde{A}_{n-1}, \tilde{C}_{n-1}$, with rank $n \geq 3$. Note that, by convention, the subscript in each case indicates the geometric dimension of the Artin group.

The Artin group $A\left(A_{n}\right)$ is well-known as the braid group on $n+1$ strings, and the automorphism groups of the braid groups were determined by Dyer and Grossman in [7]. In this paper we exploit the fact that the Artin groups of type $B, \tilde{A}$ and $\tilde{C}$ also have descriptions as braid groups (see [1]) in order to study their automorphism groups. Note that the centre of any irreducible Artin group of finite type is an infinite cyclic group, which we generally denote by $Z$. (On the other hand, the groups $A\left(\tilde{A}_{n-1}\right)$ and $A\left(\tilde{C}_{n-1}\right)$ have trivial centres.) As explained in Section 1, each of the groups $A\left(A_{n}\right) / Z, A\left(B_{n}\right) / Z, A\left(\tilde{A}_{n-1}\right)$ and $A\left(\tilde{C}_{n-1}\right)$ is a finite index subgroup of the mapping class group $\operatorname{Mod}\left(S_{n+2}\right)$ of the $(n+2)$-punctured 2-sphere $S_{n+2}$. Using this fact, and appealing to the work of Ivanov and Korkmaz on the structure of surface mapping class groups [9, 11, 12], we show:

Theorem 1. Let $C_{2}=\mathbb{Z} / 2 \mathbb{Z}$. For each $n \geq 3$, we have

(i) $\operatorname{Comm}(G) \cong \operatorname{Mod}\left(S_{n+2}\right)$ for each $G=A\left(A_{n}\right) / Z, A\left(B_{n}\right) / Z, A\left(\tilde{A}_{n-1}\right), A\left(\tilde{C}_{n-1}\right)$.

(ii) (a) (Dyer, Grossman [7]) $\operatorname{Out}\left(A\left(A_{n}\right)\right) \cong \operatorname{Out}\left(A\left(A_{n}\right) / Z\right) \cong C_{2}$

(b) $\operatorname{Out}\left(A\left(B_{n}\right)\right) \cong\left(\mathbb{Z} \rtimes C_{2}\right) \times C_{2}$ and $\operatorname{Out}\left(A\left(B_{n}\right) / Z\right) \cong C_{2} \times C_{2}$

(c) $\operatorname{Out}\left(A\left(\tilde{C}_{n-1}\right)\right) \cong \operatorname{Sym}(3) \times C_{2}$

(d) $\operatorname{Out}\left(A\left(\tilde{A}_{n-1}\right)\right) \cong D_{2 n} \times C_{2}$. 
In addition, we show that the exact sequence

$$
1 \rightarrow \operatorname{Inn}(A) \rightarrow \operatorname{Aut}(A) \rightarrow \operatorname{Out}(A) \rightarrow 1
$$

splits in each of the above cases with the exception of the case $A=A\left(\tilde{C}_{n-1}\right)$ with $n \equiv 2 \bmod 3$ (see Proposition 6$)$. In the case of $A\left(\tilde{A}_{n-1}\right)$, for example, the dihedral factor $D_{2 n}$ is realised by the group of "graph automorphisms" induced from symmetries of the Coxeter graph, which in this case is an $n$-cycle. Every Artin group admits an involution $\epsilon: s_{i} \mapsto s_{i}^{-1}$, for $i=1, . ., n$, which is never inner (because it reverses the sign of the length function $\ell: A \rightarrow \mathbb{Z}$, defined by $s_{i} \mapsto 1$ ). This accounts for the central $C_{2}$ factor in each of the outer automorphism groups above.

Note that all of the outer automorphism groups in the theorem are finite with the exception of $\operatorname{Out}\left(A\left(B_{n}\right)\right)$. The infinite cyclic factor in $\operatorname{Out}\left(A\left(B_{n}\right)\right)$ is generated by a "transvection" which multiplies each generator $s_{i}$ by some element of the center $Z$. In Section 3, we study the group of transvections of a finite type Artin group. Transvection homomorphisms exist in any Artin group with non-trivial center, but in general, they are not automorphisms. In particular, transvection automorphisms do not occur when the Artin group has abelianisation $\mathbb{Z}$, as in the case of the braid groups. However, $A\left(B_{n}\right)$ has abelianisation $\mathbb{Z} \times \mathbb{Z}$ and likewise for Artin groups of dihedral type $I_{2}(m)$ when $m$ is even. (The groups $\operatorname{Out}\left(A\left(I_{2}(m)\right)\right)$, for $m \geq 3$, were computed in [8] and are discussed in Section 3). By contrast, transvection homomorphisms appear in the abstract commensurator group of every finite type Artin group. In Proposition 11, we show that the abstract commensurator group of any finite type Artin group contains an infinitely generated free abelian group generated by transvections.

\section{Abstract commensurators and the mapping class group of a punctured sphere}

The following is a special case of Theorem 8.5A of Ivanov's survey article [9]. It is a consequence of ideas laid out in the papers of Ivanov on the mapping class groups of higher genus orientable surfaces and the work of Korkmaz [11, 12] on the complex of curves associated to an $m$-punctured sphere. For simplicity we shall write $S_{m}$ for the 2 -sphere with $m$ points removed. If $S$ is an orientable surface (without boundary), we denote by $\operatorname{Mod}(S)$ the group of all diffeomorphisms of $S$ (not necessarily respecting orientation) modulo diffeotopy.

Theorem 2 (Ivanov, Korkmaz). Let $m \geq 5$. If $\varphi: H \rightarrow K$ is an isomorphism between finite index subgroups $H, K<\operatorname{Mod}\left(S_{m}\right)$, then $\varphi$ is the restriction to $H$ of an inner automorphism of $\operatorname{Mod}\left(S_{m}\right)$ (conjugation by some element $g \in$ $\left.\operatorname{Mod}\left(S_{m}\right)\right)$.

Definition 3. Let $G$ be a group. We define the abstract commensurator group of $G$ to be

$$
\operatorname{Comm}(G)=\{\varphi: H \stackrel{\cong}{\rightrightarrows} K: H, K<G \text { finite index }\} / \sim
$$


where $\varphi \sim \psi$ if they agree on a finite index subgroup of $G$. We note that the group structure on $\operatorname{Comm}(G)$ is given by composition of isomorphisms after appropriate restriction of their domains to finite index subgroups.

Corollary 4. Let $\Gamma=\operatorname{Mod}\left(S_{m}\right), m \geq 5$, let $H<\Gamma$ denote a finite index subgroup and $N_{\Gamma}(H)$ the normalizer of $H$ in $\Gamma$. Then

(i) $\operatorname{Comm}(H) \cong \Gamma$, and

(ii) $\operatorname{Aut}(H) \cong N_{\Gamma}(H)$.

Proof. (i) There is a natural homomorphism $\Gamma \rightarrow \operatorname{Comm}(H)$ which takes $\gamma \in \Gamma$ to conjugation by $\gamma$ restricted to the finite index subgroup $H \cap \gamma^{-1} H \gamma$. It follows from Theorem 2 that this map is surjective. To show that it is injective, we observe that $\Gamma$ has trivial "virtual centre", that is, any element which centralizes a finite index subgroup is necessarily trivial. This is because any mapping class $g \in \operatorname{Mod}(S)$ is determined by its action on a finite number of isotopy classes of simple closed curves on the surface $S$, and therefore by its action on the $n$-th powers $T_{i}^{n}$ of the corresponding Dehn twists for any choice of $n$. Given a finite index subgroup $K<\Gamma$ centralized by $g$ we can always find a sufficiently large $n$ such that $K$ contains all $T_{i}^{n}$. Then $g$ is determined by its action on $K$ and so must be the identity in $\Gamma$.

(ii) There is now an obvious homomorphism $\operatorname{Aut}(H) \rightarrow \Gamma$ which factors through the inverse of the isomorphism just described, and realizes each automorphism of $H$ as conjugation by an element of $\Gamma$. By the preceding argument this map is injective. Its image is precisely $N_{\Gamma}(H)$.

\section{Some interesting finite index subgroups of $\operatorname{Mod}\left(S_{m}\right)$ and their automorphisms}

Let $\operatorname{Sym}(m)$ denote the group of permutations of the set $\{1,2, . ., m\}$, for $m \geq 1$. If $k<m$ then we consider $\operatorname{Sym}(k)$ as a subgroup of $\operatorname{Sym}(m)$ consisting of those permutations which fix the subset $\{k+1, . ., m\}$. Notice that each mapping class of the $m$-punctured sphere $S_{m}$ induces a permutation of the punctures. Moreover, the mapping class group acts on the orientation class of the surface. Thus we have a surjective group homomorphism $\pi: \operatorname{Mod}\left(S_{m}\right) \rightarrow \operatorname{Sym}(m) \times C_{2}$ with kernel the group of pure orientation preserving mapping classes. Note that, strictly speaking, the definition of $\pi$ depends on a labelling of the punctures by the numbers 1 through $m$.

Notation. Fix $n \geq 3$. We shall write $\Gamma=\operatorname{Mod}\left(S_{n+2}\right)$ and $\Gamma_{A}, \Gamma_{B}, \Gamma_{\tilde{C}}$ respectively, for the finite index subgroup of $\Gamma$ generated by those orientation preserving diffeomorphisms which fix the last 1,2, 3 punctures of $S_{n+2}$ respectively. These three subgroups are, in other words, the preimages under $\pi$ of the subgroups $\operatorname{Sym}(k)<\operatorname{Sym}(n+2) \times C_{2}$, with $k=n+1, n, n-1$ respectively. In particular we have $\left[\Gamma: \Gamma_{A}\right]=2(n+2),\left[\Gamma: \Gamma_{B}\right]=2(n+1)(n+2)$ and $\left[\Gamma: \Gamma_{\tilde{C}}\right]=2 n(n+1)(n+2)$. 
It is well known that the $(n+1)$-string braid group, $A\left(A_{n}\right)$, is isomorphic to the orientation preserving mapping class group of the $(n+1)$-punctured disk relative to the boundary of the disk. (That is, diffeotopies are required in this case to fix the boundary of the disk pointwise). The centre $Z$ of this mapping class group is generated by the Dehn twist about a curve parallel to the boundary, and is precisely the kernel of the natural homomorphism to the mapping class group $\Gamma=\operatorname{Mod}\left(S_{n+2}\right)$ (induced by inclusion of the punctured disk in $\left.S_{n+2}\right)$. Thus we may realise the group $A\left(A_{n}\right) / Z$ as the finite index subgroup $\Gamma_{A}$ of $\Gamma$. Likewise, the group $A\left(B_{n}\right)$ may be identified with the subgroup of $A\left(A_{n}\right)$ fixing the last string, so $A\left(B_{n}\right) / Z$ is isomorphic to $\Gamma_{B}$.

It is less well-known that the Artin group $A\left(\tilde{C}_{n-1}\right)$ is also isomorphic to a subgroup of the braid group. To describe this subgroup, renumber the punctures so that $\Gamma_{\tilde{C}}$ fixes $x_{1}, x_{n+1}, x_{n+2}$ and let $\widehat{\Gamma}_{\tilde{C}}$ be the inverse image of $\Gamma_{\tilde{C}}$ in $A\left(A_{n}\right)$. (Thus $\widehat{\Gamma}_{\tilde{C}}$ is a "2-pure" braid group.) Forgetting strings 2 through $n$ gives a homomorphism $\widehat{\Gamma}_{\tilde{C}} \rightarrow A\left(A_{1}\right) \cong \mathbb{Z}$ which counts the twisting between the first and last string. As shown in [1], the Artin group $A\left(\tilde{C}_{n-1}\right)$ can be identified with the kernel of this homomorphism. We can picture the kernel as the subgroup of braids in which the first and last strings have been pulled straight so they cannot interact. It is easy to see that this subgroup intersects the center $Z$ (of $A\left(A_{n}\right)$ ) trivially and together they generate $\widehat{\Gamma}_{\tilde{C}}$. It follows that $A\left(\tilde{C}_{n-1}\right)$ is isomorphic to $\Gamma_{\tilde{C}}$.

In summary, we have

- $\Gamma_{A} \cong A\left(A_{n}\right) / Z$,

- $\Gamma_{B} \cong A\left(B_{n}\right) / Z$, and

- $\Gamma_{\tilde{C}} \cong A\left(\tilde{C}_{n-1}\right)$.

These isomorphisms may be described explicitly as follows. Distribute the punctures $x_{i}$ evenly along half the equator in the order $x_{n+2}, x_{1}, x_{2}, . ., x_{n}, x_{n+1}$ so that $x_{n+1}$ and $x_{n+2}$ are antipodal (see Figure 2). Then the $i$ th standard generator of $A\left(A_{n}\right)$ maps to the positive braid twist $\sigma_{i}$ exchanging $x_{i}$ with $x_{i+1}$. The $i$ th standard generator of $A\left(B_{n}\right)$ maps to $\sigma_{n}^{2}$ if $i=n$ and $\sigma_{i}$ otherwise. The $i$ th standard generator of $A\left(\tilde{C}_{n-1}\right)$ maps to $\sigma_{i}^{2}$ if $i \in\{1, n\}$ and $\sigma_{i}$ if $i \in\{2, . ., n-1\}$.

Proposition 5. (i): $\operatorname{Comm}\left(\Gamma_{A}\right) \cong \operatorname{Comm}\left(\Gamma_{B}\right) \cong \operatorname{Comm}\left(\Gamma_{\tilde{C}}\right) \cong \Gamma$,

(ii): there exist short exact sequences

$$
\begin{aligned}
& 1 \rightarrow \Gamma_{A} \rightarrow \operatorname{Aut}\left(\Gamma_{A}\right) \rightarrow C_{2} \rightarrow 1 \\
& 1 \rightarrow \Gamma_{B} \rightarrow \operatorname{Aut}\left(\Gamma_{B}\right) \rightarrow C_{2} \times C_{2} \rightarrow 1 \\
& 1 \rightarrow \Gamma_{\tilde{C}} \rightarrow \operatorname{Aut}\left(\Gamma_{\tilde{C}}\right) \rightarrow \operatorname{Sym}(3) \times C_{2} \rightarrow 1
\end{aligned}
$$

Proof. Let $H$ denote one of $\Gamma_{A}, \Gamma_{B}$, or $\Gamma_{\tilde{C}}$, and $P$ the set of 1,2 or 3 punctures fixed by all elements of $H$. It is easily checked that $N_{\Gamma}(H)$ is exactly the group of all mapping classes (orientation preserving or not) which leave $P$ setwise invariant. It follows that $N_{\Gamma}(H) / H$ is a direct product of $C_{2}=\Gamma / \Gamma^{+}$with the group $\operatorname{Sym}(P)$ of permutations of the set $P$. Statements (i) and (ii) now follow immediately from the corresponding statements in Corollary 4. 


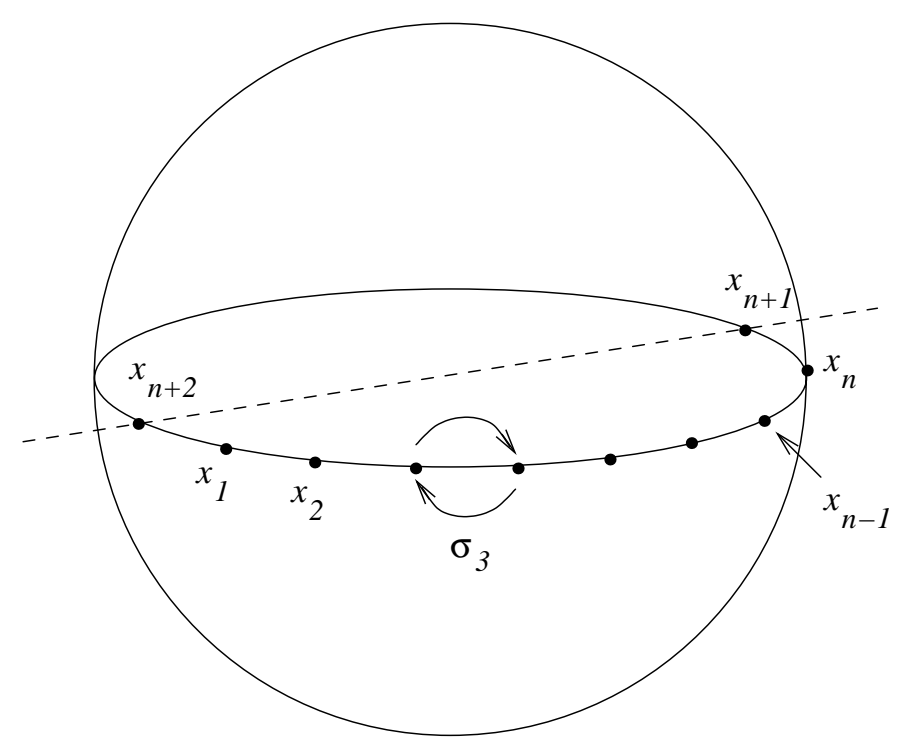

FiguRE 2. Finite index subgroups of $\Gamma=\operatorname{Mod}\left(S_{n+2}\right)$ isomorphic to $A\left(A_{n}\right) / Z, A\left(B_{n}\right) / Z$ and $A\left(\tilde{C}_{n-1}\right), n \geq 3$, fix the subsets $\left\{x_{n+2}\right\},\left\{x_{n+1}, x_{n+2}\right\}$ and $\left\{x_{1}, x_{n+1}, x_{n+2}\right\}$ respectively.

It is easy to see, with the aid of Figure 2, that the short exact sequences in the proposition above are split in the cases $\Gamma_{A}$ and $\Gamma_{B}$. The group $C_{2} \times C_{2}$ may be generated by a reflection in the equatorial plane and a rotation about an axis in the equatorial plane which exchanges the points $x_{n+1}$ and $x_{n+2}$. These diffeomorphisms leave $\Gamma_{B}$ invariant. Only the reflection leaves $\Gamma_{A}$ invariant.

For $\Gamma_{\tilde{C}}$, the exact sequence splits providing $n+2 \equiv 0,2 \bmod 3$. To see this, think of $S_{n+2}$ as a sphere with $3 k$ punctures arranged symmetrically along the equator and, if $n+2 \equiv 2(3)$, the remaining two punctures at the north and south poles. Then there is an orientation-preserving action of $\operatorname{Sym}(3)$ on $S_{n+2}$ generated by an order three rotation $\rho$ about the vertical axis through the poles and an order two rotation $\eta$ about a horizontal axis through one ( $k$ odd) or two ( $k$ even) punctures at the equator. If $x$ is a puncture fixed by $\eta$, then the orbit of $x$ under $\rho$ is preserved by $\operatorname{Sym}(3)$, hence for an appropriate numbering of the punctures (namely, so that $P=\left\{x, \rho(x), \rho^{2}(x)\right\}$ ) the action of $\operatorname{Sym}(3)$ lies in $\operatorname{Aut}\left(\Gamma_{\tilde{C}}\right)$ and is faithful on $P$. This splits the $\operatorname{Sym}(3)$ factor. The $C_{2}$ factor is realized by reflection through the equatorial plane.

Proposition 6. If $\Gamma_{\tilde{C}}=A\left(\tilde{C}_{n-1}\right)$ with $n+2 \equiv 1 \bmod 3$, then Aut $\left(\Gamma_{\tilde{C}}\right)$ does not contain a subgroup isomorphic to $\operatorname{Sym}(3)$. In this case, the exact sequence for $\Gamma_{\tilde{C}}$ in the previous proposition does not split.

Proof. Suppose $\operatorname{Aut}\left(\Gamma_{\tilde{C}}\right) \subset \Gamma$ contains a subgroup $K$ isomorphic to $\operatorname{Sym}(3)$ and let $H$ be the subgroup of $K$ generated by a 3-cycle. Then $H$ is a subgroup of $\operatorname{Mod}\left(S_{n+2}\right)$, hence $H$ acts by permutations on the set of punctures $\left\{x_{1}, \ldots x_{n+2}\right\}$. 
Every $H$ orbit consists of either 1 or 3 punctures. Since $n+2 \equiv 1(3)$, the number of fixed points of $H$ must also be congruent to $1 \bmod 3$. If $H$ fixes 4 or more punctures, then it lies in a subgroup of $\Gamma$ isomorphic to $\Gamma_{\tilde{C}}$ which is impossible since $\Gamma_{\tilde{C}}$ is torsion-free (since, by [1], it is a subgroup of the braid group on $n+1$ strings which is known to be torsion free).

Thus $H$ must have a unique fixed puncture. Since $H$ is normal in $K$, all of $K$ must fix this puncture and hence $K$ lies in a subgroup of $\Gamma$ isomorphic to $\Gamma_{A}$. By a theorem of Bestvina, [2] Thm 4.5, every finite subgroup of $\Gamma_{A}$ is cyclic, so we arrive at a contradiction.

It has been observed by several authors [1][6][10] that there exists a semidirect product decomposition

$$
A\left(B_{n}\right) \cong A\left(\tilde{A}_{n-1}\right) \rtimes \mathbb{Z} .
$$

(See [5] for further discussion of this decomposition.) The Coxeter graph of $A\left(\tilde{A}_{n-1}\right)$ is an $n$-cycle and the generator of the cyclic factor in the semi-direct product acts on $A\left(\tilde{A}_{n-1}\right)$ via an order $n$ rotation of this graph. The centre of $A\left(B_{n}\right)$ is the subgroup $n \mathbb{Z}$ of the cyclic factor. Thus we also have

$$
\Gamma_{B} \cong A\left(\tilde{A}_{n-1}\right) \rtimes \mathbb{Z} / n \mathbb{Z} .
$$

Here we interpret $A\left(\tilde{A}_{n-1}\right)$ as the subgroup of $\Gamma_{B}$ consisting of mapping classes of "zero angular momentum" about the axis through the two fixed punctures. More precisely, suppose that the punctures of $S_{n+2}$ are arranged so that $x_{n+1}$ and $x_{n+2}$ are placed at the north and south pole respectively, and the remaining points $x_{1}, . ., x_{n}$ are equally spaced (in that cyclic order) around the equator, see figure 3. Define $\Gamma_{\tilde{A}}$ to be the subgroup of $\Gamma$ generated by the braid twists $\sigma_{i}$ which exchange the points $x_{i}$ and $x_{i+1}$, for $i=1, . ., n$, indices taken mod $n$. The isomorphism $A\left(\tilde{A}_{n-1}\right) \cong \Gamma_{\tilde{A}}$ is given by sending the $i$ th standard generator of $A\left(\tilde{A}_{n-1}\right)$ to $\sigma_{i}$. Note that $\Gamma_{\tilde{A}}$ is a finite index subgroup of $\Gamma$ of index $\left[\Gamma: \Gamma_{\tilde{A}}\right]=$ $n\left[\Gamma: \Gamma_{B}\right]=2 n(n+1)(n+2)$. In particular, $\operatorname{Comm}\left(\Gamma_{\tilde{A}}\right) \cong \Gamma$.

Proposition 7. Let $\Gamma_{\tilde{A}} \cong A\left(\tilde{A}_{n-1}\right)$ be as above. Then $N_{\Gamma}\left(\Gamma_{\tilde{A}}\right)=N_{\Gamma}\left(\Gamma_{B}\right)$. Consequently $\operatorname{Aut}\left(\Gamma_{\tilde{A}}\right) \cong \operatorname{Aut}\left(\Gamma_{B}\right)$ and there is an exact sequence

$$
1 \rightarrow \Gamma_{\tilde{A}} \rightarrow \operatorname{Aut}\left(\Gamma_{\tilde{A}}\right) \rightarrow D_{2 n} \times C_{2} \rightarrow 1
$$

where $D_{2 n}$ denotes the dihedral group of order $2 n$. Moreover, a splitting of this sequence is given by realizing the group of outer automorphisms as the direct product of the group of graph automorphisms of $A\left(\tilde{A}_{n-1}\right)$ with the group of order 2 generated by the inversion $\epsilon$ which inverts each standard generator.

Proof. We suppose that the punctures of $S_{n+2}$ are arranged as shown in Figure 3. The outer automorphism group of $\Gamma_{B}$ may then be generated by the reflection in the equatorial plane and a rotation exchanging the two poles (this gives a splitting of the exact sequence of Proposition 5 slightly different to the 


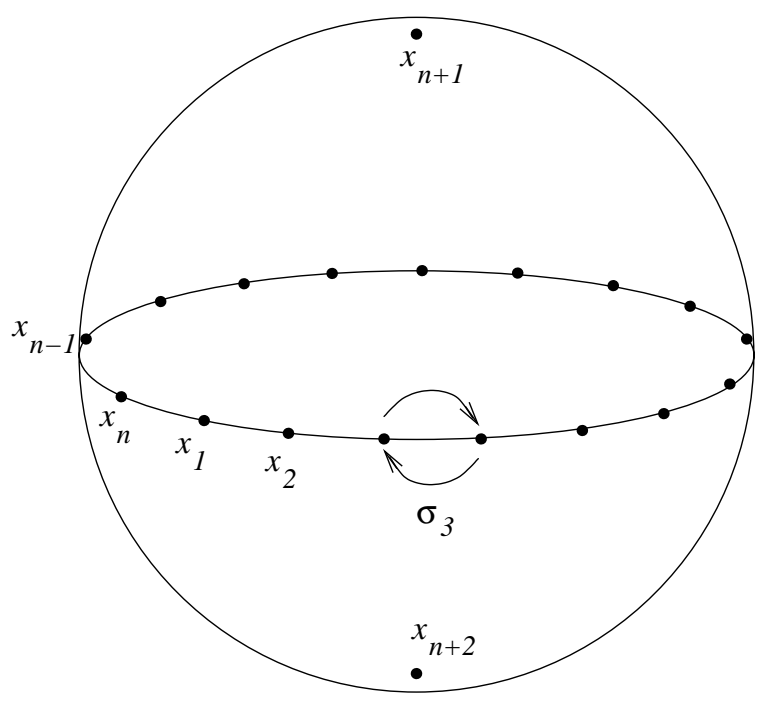

Figure 3. The finite index subgroup $\Gamma_{\tilde{A}}$ of $\Gamma=\operatorname{Mod}\left(S_{n+2}\right)$ isomorphic to $A\left(\tilde{A}_{n-1}\right)$.

one previously mentioned). It follows easily that $\Gamma_{\tilde{A}}$ is invariant by these mapping classes, and hence a characteristic subgroup of $\Gamma_{B}$. In particular, we have $N_{\Gamma}\left(\Gamma_{B}\right)<N_{\Gamma}\left(\Gamma_{\tilde{A}}\right)$.

Let $P=\left\{x_{n+1}, x_{n+2}\right\}$. To prove that $N_{\Gamma}\left(\Gamma_{\tilde{A}}\right)=N_{\Gamma}\left(\Gamma_{B}\right)$ it suffices now to check that every mapping class which normalizes $\Gamma_{\tilde{A}}$ leaves $P$ invariant. But if $g \in N_{\Gamma}\left(\Gamma_{\tilde{A}}\right)$ then $\pi(g)$ normalizes $\pi\left(\Gamma_{\tilde{A}}\right)=\operatorname{Sym}(n) \times C_{2}$, and this is just to say that $g$ induces a symmetry of the puncture set which leaves $P$ invariant, as required.

The remaining statements are now easily deduced from Corollary 4 and Proposition 5 .

In summary, Propositions 5 and 7 give us explicitly the automorphism groups of $A\left(A_{n}\right) / Z, A\left(B_{n}\right) / Z, A\left(\tilde{A}_{n-1}\right)$, and $A\left(\tilde{C}_{n-1}\right)$, for all $n \geq 3$, as well as the fact that the abstract commensurator group is in each case isomorphic to $\operatorname{Mod}\left(S_{n+2}\right)$. This proves Theorem 1 with the exception of the first isomorphism in each of part (ii)(a) and (b). In the next section we shall recover the group $\operatorname{Aut}\left(A\left(B_{n}\right)\right)$ from the above result on $\operatorname{Aut}\left(A\left(B_{n}\right) / Z\right)$. In a similar fashion, the result of $[7]$ on automorphisms of the braid group is easily recovered from the computation of $\operatorname{Aut}\left(A\left(A_{n}\right) / Z\right)$. These steps will complete the proof of Theorem 1.

\section{Transvections and automorphisms of $A\left(B_{n}\right)$}

Let $H$ be a group with nontrivial centre $Z$. By a transvection of $H$ we mean a homomorphism $T_{\lambda}: H \rightarrow H$ given by $T_{\lambda}(x)=x \lambda(x)$, where $\lambda$ denotes a 
function $H \rightarrow Z$. The fact that $T_{\lambda}$ is a homomorphism requires that $\lambda$ is also a homomorphism, and it is easily checked that the composite of two transvections is again a transvection. We note, however, that transvections are typically not automorphisms. It is easily shown that a transvection is an automorphism (resp. injective, resp. surjective) if and only if its restriction to the centre $Z$ is an automorphism (resp. injective, resp. surjective).

Let $\operatorname{Tv}(H)$ denote the group of automorphisms of $H$ which are transvections. Then, almost by definition, we have an exact sequence

$$
1 \rightarrow \operatorname{Tv}(H) \rightarrow \operatorname{Aut}(H) \rightarrow \operatorname{Aut}(H / Z)
$$

Note that $\operatorname{Inn}(H) \cong H / Z$ always maps isomorphically onto $\operatorname{Inn}(H / Z)$. In general there is an issue, however, as to whether every outer automorphism of $H / Z$ lifts to an automorphism of $H$, and as to whether the extension splits.

In the case of an irreducible finite type Artin group $A$, the extension splits at least over a finite index subgroup $A u t^{*}(A / Z)$ of $A u t(A / Z)$ defined as follows. Let $\ell: A \rightarrow \mathbb{Z}$ be the length homomorphism which maps each generator of $A$ to 1. Recall that the centre $Z$ of $A$ is infinite cyclic. Let $\zeta$ be a generator of $Z$ and let $d=\ell(\zeta)$. The length homomorphism on $A$ descends to a "length homomorphism" $\bar{\ell}: A / Z \rightarrow \mathbb{Z} / d \mathbb{Z}$. Define $A u t^{*}(A)$, respectively $A u t^{*}(A / Z)$, to be the group of length preserving or reversing automorphisms, that is, automorphisms $\alpha$ such that $\ell \circ \alpha= \pm \ell$ (respectively $\bar{\ell} \circ \alpha= \pm \bar{\ell}$ ).

Proposition 8. Suppose $A$ is an irreducible Artin group of finite type. Then the natural map $\pi: \operatorname{Aut}^{*}(A) \rightarrow \operatorname{Aut}^{*}(A / Z)$ is an isomorphism. If, in addition, the abelianization of $A$ is infinite cyclic (i.e., $A$ is not of type $I_{2}(2 m), B_{n}$, or $\left.F_{4}\right)$, then $\operatorname{Tv}(A)$ is trivial and $\operatorname{Aut}(A) \cong \operatorname{Aut}^{*}(A / Z)$.

Proof. Note that a non-trivial transvection $T_{\lambda}$ of $A$ can never be length preserving, and can only be length reversing if $d=2\left(\right.$ and $\left.\lambda(\cdot)=\zeta^{-\ell(\cdot)}\right)$. Since $d>2$ for all irreducible finite type Artin groups we conclude that $\operatorname{ker}(\pi)=$ $\operatorname{Tv}(A) \cap A u t^{*}(A)$ is trivial.

For any length preserving $\alpha \in A u t^{*}(A / Z)$, we define a lift $\hat{\alpha} \in A u t^{*}(A)$ as follows. (The lift for length reversing automorphisms is defined analogously). By definition, for any $a \in A, \alpha(a Z)=b Z$ for some $b \in A$ with $\ell(b) \equiv \ell(a) \bmod$ $d$. Since $Z$ is generated by an element of length $d$, the coset $b Z$ contains a unique representative $b^{\prime}$ satisfying $\ell\left(b^{\prime}\right)=\ell(a)$. Define $\hat{\alpha}(a)=b^{\prime}$. To verify that $\hat{\alpha}$ is a homomorphism, note that if $\hat{\alpha}\left(a_{1}\right)=b_{1}$ and $\hat{\alpha}\left(a_{2}\right)=b_{2}$, then

$$
\begin{aligned}
\alpha\left(a_{1} a_{2} Z\right) & =\alpha\left(a_{1} Z\right) \cdot \alpha\left(a_{2} Z\right)=b_{1} Z \cdot b_{2} Z=b_{1} b_{2} Z, \\
\ell\left(a_{1} a_{2}\right) & =\ell\left(a_{1}\right)+\ell\left(a_{2}\right)=\ell\left(b_{1}\right)+\ell\left(b_{2}\right)=\ell\left(b_{1} b_{2}\right),
\end{aligned}
$$

so it follows that $\hat{\alpha}\left(a_{1} a_{2}\right)=b_{1} b_{2}$. Moreover, it is straightforward to verify that if $\alpha_{1}$ and $\alpha_{2}$ are two elements of $\operatorname{Aut}^{*}(A / Z)$, then $\alpha_{1} \circ \alpha_{2}$ lifts to $\hat{\alpha}_{1} \circ \hat{\alpha}_{2}$. In particular, the lift of an automorphism is also an automorphism. Thus, $\alpha \mapsto \hat{\alpha}$ defines a section for the projection $\pi$, and $\pi$ is an isomorphism.

If the abelianization of $A$ is infinite cyclic, then the length homomorphism $\ell$ can be identified with the abelianization map $A \rightarrow A^{\text {ab }} \cong \mathbb{Z}$. Hence every 
automorphism of $A$ is either length preserving or length reversing. The second statement of the proposition follows.

In the case of $A=A\left(A_{n}\right)$, it follows from Proposition 5 that $A u t^{*}(A / Z)=$ $\operatorname{Aut}(A / Z) \cong \Gamma_{A} \rtimes C_{2}$ which, combined with Proposition 8, yields the main result of [7], that $\operatorname{Out}\left(A\left(A_{n}\right)\right)=C_{2}$.

We remark, however, that while $\operatorname{Aut}(A)=A u t^{*}(A) \cong A u t^{*}(A / Z)$ when $A$ has infinte cyclic abelianization, it is not always the case that $\operatorname{Aut}(A) \cong \operatorname{Aut}(A / Z)$ (or equivalently that $\operatorname{Out}(A) \cong \operatorname{Out}(A / Z)$ ). In particular, for $A$ of type $I_{2}(m)$ with $m$ odd, $A / Z \cong C_{2} * C_{m}$, and $\operatorname{Out}(A / Z)$ is isomorphic to the group of units of $\mathbb{Z} / m \mathbb{Z}$, while $\operatorname{Out}(A)=C_{2}$.

In the case of an Artin group whose abelianization is not infinite cyclic, the situation is more complicated.

Proposition 9. If $A$ is an irreducible Artin group of type $I_{2}(2 n), B_{n}, n \geq 3$, or $F_{4}$, then $\operatorname{Tv}(A) \cong \mathbb{Z}$. If $A$ is of type $I_{2}(4) \equiv B_{2}$ then $\operatorname{Tv}(A) \cong D_{\infty}$. Moreover, in all these cases $A u t(A)$ contains $\operatorname{Tv}(A) \rtimes A u t^{*}(A)$ as a finite index subgroup.

Proof. We refer the reader to [3] and [4] for descriptions of the Coxeter graphs, and central elements respectively, for each of the irreducible finite type Artin groups. In each case listed above, the abelianization of $A$ is $\mathbb{Z} \times \mathbb{Z}$, and the generator $\zeta$ of $Z$ maps to an element $(r, s) \in \mathbb{Z} \times \mathbb{Z}$, where $(r, s)=(n, n)$ for type $I_{2}(2 n),(12,12)$ for type $F_{4}$, and $(n(n-1), n)$ for type $B_{n}$. Any homomorphism $\lambda: A \rightarrow Z$ can be obtained by composing the abelianisation homomorphism with a map $\mathbb{Z} \times \mathbb{Z} \rightarrow Z$ given by $(r, s) \mapsto \zeta^{p r+q s}$ for a pair of integers $p, q \in \mathbb{Z}$. The associated transvection then satisfies $T_{\lambda}(\zeta)=\zeta^{k}$ where $k=1+(p+q) n$, or $1+(p(n-1)+q) n$, depending on the case $\left(n=12\right.$ for type $\left.F_{4}\right)$. On the other hand $T_{\lambda}$ is an automorphism if and only if $k= \pm 1$. When $n \geq 3$ this is only possible if $k=1$ and $p+q=0$, or $(p(n-1)+q)=0$, respectively. This allows exactly an infinite cyclic group of transvection automorphisms (all of which act by the identity on $Z$ ).

In the remaining case, type $I_{2}(4) \equiv B_{2}$, we have the constraint $k=1+2(p+$ $q)= \pm 1$, and so $p+q=0$ or -1 . We note that $A=A\left(I_{2}(4)\right)$ is given by the presentation $\langle a, b \mid a b a b=b a b a\rangle$, where $\zeta=a b a b$. A transvection of the second type $(p=0, q=-1)$ is realized by the automorphism $T_{0}: a \mapsto a$ and $b \mapsto(a b a)^{-1}$. Since in general we have

$$
T_{\lambda} \circ T_{\mu}= \begin{cases}T_{\lambda+\mu} & \text { if } T_{\lambda}(\zeta)=\zeta \\ T_{\lambda-\mu} & \text { if } T_{\lambda}(\zeta)=\zeta^{-1}\end{cases}
$$

it is easily checked that $\operatorname{Tv}(A)$ is an infinite dihedral group with $T_{0}$ acting as a direction reversing involution.

The subgroup $A u t^{*}(A / Z)$ is finite index in $\operatorname{Aut}(A / Z)$ (since $\bar{\ell}$ takes values in the finite group $\mathbb{Z} / d \mathbb{Z}$ and $A / Z$, being finitely generated, admits only a finite number of homomorphisms to $\mathbb{Z} / d \mathbb{Z})$. Hence the inverse image $\pi^{-1}\left(A u t^{*}(A / Z)\right)$ $<\operatorname{Aut}(A)$ is a finite index subgroup of $\operatorname{Aut}(A)$. By the previous proposition, this subgroup splits as a semi-direct product $T v(A) \rtimes A u t^{*}(A)$. 
For $A$ of type $I_{2}(m)$, we have the following presentation:

$$
A\left(I_{2}(m)\right)=\langle a, b \mid \underbrace{a b a \ldots}_{m}=\underbrace{b a b \ldots}_{m}\rangle .
$$

In this case the outer automorphism groups were computed in [8]:

$$
\begin{aligned}
& \operatorname{Out}\left(A\left(I_{2}(m)\right) \cong C_{2},\right. \\
& \operatorname{Out}\left(A\left(I_{2}(m)\right) \cong(\mathbb{Z} \rtimes\langle\gamma\rangle) \times C_{2} \cong D_{\infty} \times C_{2}, m \text { odd }\right. \text { even }
\end{aligned}
$$

where $\gamma$ denotes the graph involution $\gamma: a \leftrightarrow b$, and the $C_{2}$ factor in each case is generated by the inversion automorphism $\epsilon: a \mapsto a^{-1}, b \mapsto b^{-1}$. In the case $m$ even, the infinite cyclic factor $\mathbb{Z}$ is generated by the automorphism $\eta: a \mapsto a b a$ and $b \mapsto a^{-1}=b(a b)^{-1}$, and contains the group of transvections as a subgroup of index $m / 2$, when $m \geq 6$. (Note that $\eta$ fixes both elements $a b$ and $b a$, and that the centre is generated by $\left.(a b)^{m / 2}=(b a)^{m / 2}\right)$. It follows that, in the case $A=A\left(I_{2}(m)\right)$ with $m \geq 6$ even, the subgroup $T v(A) \rtimes A u t^{*}(A)$ has index $m / 2$ in the entire automorphism group. This behaviour seems to be repeated in the case $A=A\left(B_{n}\right)$, for $n \geq 3$, where, as we will see below, $\pi^{-1}\left(A u t^{*}(A / Z)\right)$ is a subgroup of index 2 in $\operatorname{Aut}(A)$. The type $I_{2}(4) \equiv B_{2}$ Artin group is however exceptional in this regard. In this case the group of transvections is generated by $\eta^{2}$ and the element $T_{0}=\epsilon \circ \eta \circ \gamma$, from which it follows that $T v(A) \rtimes A u t^{*}(A)$ is the whole automorphism group. We do not know what the index is (or whether the subgroup is proper) in the case of $A\left(F_{4}\right)$.

Proposition 10. Let $n \geq 3$ and $A=A\left(B_{n}\right)$. Then

$$
\begin{aligned}
& \operatorname{Aut}(A) \cong\left(\Gamma_{B} \times T v(A)\right) \rtimes\left(C_{2} \times C_{2}\right) \\
& \operatorname{Out}(A) \cong\left(\mathbb{Z} \rtimes C_{2}\right) \times C_{2} \cong D_{\infty} \times C_{2}
\end{aligned}
$$

Proof. We first show that the map $\phi: \operatorname{Aut}(A) \rightarrow \operatorname{Aut}(A / Z)$ is surjective and splits. Recall from Section 2 that $A / Z \cong \Gamma_{B} \cong \Gamma_{\tilde{A}} \rtimes \mathbb{Z} / n \mathbb{Z}$. It is clear from the proof of Proposition 7 that elements of $A u t\left(\Gamma_{B}\right)$ act on this semidirect product by leaving the characteristic subgroup $\Gamma_{\tilde{A}}$ invariant and by mapping the generator of the cyclic factor $\mathbb{Z} / n \mathbb{Z}$ either to itself or its inverse. Any such automorphism lifts uniquely to an automorphism of $A\left(B_{n}\right) \cong \Gamma_{\tilde{A}} \rtimes \mathbb{Z}$. Thus we have a section to the map $\phi$.

By Proposition 5, this proves that

$$
\operatorname{Aut}(A) \cong \operatorname{Tv}(A) \rtimes \operatorname{Aut}\left(\Gamma_{B}\right) \cong \operatorname{Tv}(A) \rtimes\left(\Gamma_{B} \rtimes\left(C_{2} \times C_{2}\right)\right) .
$$

A direct check shows that inner automorphisms, $\Gamma_{B}$, commute with transvections, and the first statement of the proposition follows.

We claim that the $C_{2}$ factor generated by the inversion $\epsilon$ also commutes with transvections. This follows from the fact that $\epsilon$ restricts to a length reversing automorphism of the center $Z$ and hence takes the generator $\zeta$ of $Z$ to $\zeta^{-1}$. For any transvection $T$ and any generator $s_{i}, T\left(s_{i}\right)=s_{i} \zeta^{k}$ for some $k$, so

$$
\epsilon \circ T \circ \epsilon\left(s_{i}\right)=\epsilon \circ T\left(s_{i}^{-1}\right)=\epsilon\left(s_{i}^{-1} \zeta^{-k}\right)=s_{i} \zeta^{k}=T\left(s_{i}\right) .
$$


By Proposition $9, \operatorname{Tv}(A) \cong \mathbb{Z}$ and the second claim of the proposition now follows.

Remark. The $\mathbb{Z}$ factor in the decomposition $A\left(B_{n}\right)=\Gamma_{\tilde{A}} \rtimes \mathbb{Z}$ is generated by the element $\delta=s_{1} s_{2} \ldots s_{n}$ of $A\left(B_{n}\right)$ and it acts on $\Gamma_{\tilde{A}}$ via rotation of the $\tilde{A}$ graph. These rotations thus become inner automorphisms in $\operatorname{Aut}\left(A\left(B_{n}\right)\right)$. The remaining graph automorphisms (the reflections) give rise to the first of the two $C_{2}$ factors in $\operatorname{Aut}\left(A\left(B_{n}\right)\right)$, the second $C_{2}$ factor being generated by the inversion $\epsilon$. A generator of the first $C_{2}$ factor is represented by the automorphism $\tau$ defined by

$$
\begin{aligned}
\tau\left(s_{i}\right) & =s_{n-i}, i=1, \ldots, n-1 \\
\tau(\delta) & =\delta^{-1}
\end{aligned}
$$

and hence $\tau\left(s_{n}\right)=\tau\left(\left(s_{1} \ldots s_{n-1}\right)^{-1} \delta\right)=\left(\delta s_{n-1} \ldots s_{1}\right)^{-1}$. In particular, $\tau$ does not lie in $T v(A) \rtimes A u t^{*}(A)$ (as long as $n \geq 3$ ), and this latter group is index 2 in the full automorphism group. (By contrast, when $A$ is of type $B_{2} \equiv I_{2}(4)$, the involution $\tau$ just described is exactly the exceptional transvection $T_{0}=\epsilon \circ \eta \circ \gamma$ already mentioned in the preceding discussion).

If $H$ is a group whose centre $Z$ is a finite rank free abelian group, the injective transvections which are not automorphisms of $H$ have as image a proper finite index subgroup of $H$ and therefore represent infinite order elements of $\operatorname{Comm}(H)$ (not equivalent to automorphisms). In the case of a finite type Artin group, this implies that the abstract commensurator group must be large.

Proposition 11. Let $A$ be any finite type Artin group. Then $\operatorname{Comm}(A)$ contains an infinitely generated free abelian subgroup generated by transvections.

Proof. It suffices to prove the proposition for irreducible $A$. Let $\ell: A \rightarrow \mathbb{Z}$ be the length homomorphism. Let $\zeta$ be the generator of the centre $Z$ with positive length $d=\ell(\zeta)$. Then for every $m \in \mathbb{Z}$, there is a homomorphism $\lambda_{m}: A \rightarrow Z$ defined by $\lambda_{m}(a)=\zeta^{m \ell(a)}$. The associated transvection takes $a$ to $a \zeta^{m \ell(a)}$. In particular, it takes $\zeta$ to $\zeta^{m d+1}$. We denote this transvection by $T_{m d+1}$.

It is straightforward to verify that $T_{m d+1} \circ T_{k d+1}=T_{(m d+1)(k d+1)}$. Thus, $T_{m d+1}$ and $T_{k d+1}$ are commuting elements of $\operatorname{Comm}(A)$ and to find an infinitely generated free abelian subgroup of $\operatorname{Comm}(A)$, it suffices to find an infinite sequence of integers $\left\{n_{i}\right\}$ which are relatively prime and satisfy $n_{i} \equiv 1 \bmod d$. This can be done inductively by setting $n_{1}=1+d$ and $n_{i+1}=1+d\left(n_{1} n_{2} \ldots n_{i}\right)$. The transvections $T_{n_{i}}$ are then linearly independent.

\section{Acknowledgement}

The first author would like to thank the Institut de Mathematiques de Bourgogne for their hospitality during the development of this paper. 


\section{References}

[1] D. Allcock, Braid pictures for Artin groups, Trans. Amer. Math. Soc. 354 (2002), 34553474 .

[2] M. Bestvina, Non-positively curved aspects of Artin groups of finite type, Geometry \& Topology 3 (1999), 269-302.

[3] N. Bourbaki, Groupes et algèbres de Lie, Chaps 4-6, Hermann, Paris, 1968.

[4] E. Brieskorn and K. Saito, Artin-Gruppen und Coxeter-Gruppen, Invent. Math. 17 (1972), 245-271.

[5] R. Charney and D. Peifer, The $K(\pi, 1)$ conjecture for the affine braid groups, Comm. Math. Helv. 78 (2003), 584-600.

[6] T. tom Dieck, Categories of rooted cylinder ribbons and their representations, J. Reine Angew. Math. 494 (1998), 35-63.

[7] J.L. Dyer and E.K. Grossman, The automorphism groups of the braid groups, Amer. J. Math. 103 (1981), 1151-1169.

[8] N.D. Gilbert, J. Howie, V. Metaftsis and E. Raptis, Tree actions of automorphism groups, J. Group Theory 3 (2000), 213-223.

[9] N.V. Ivanov, Mapping Class Groups, "Handbook of Geometric Topology" ed. R.J. Daverman and R.B. Sher, Elsevier, 2002.

[10] R. Kent and D. Peifer, A geometric and algebraic description of the annular braid groups, Int. Jour. Alg. Comp. 12 (2002), 85-97.

[11] M. Korkmaz, Complexes of curves and mapping class groups, Ph.D. Thesis, Michigan State University, 1996.

[12] _ Automorphisms of complexes of curves on punctured spheres and on punctured tori, Topology and its Applications 95 (1999), 85-111.

Department of Mathematics, Brandeis University, Mail Stop 050, Waltham, MA 02454

E-mail address: charney@brandeis.edu

I.M.B.(UMR 5584 DU CNRS), Université DE Bourgogne, B.P. 47 870, 21078 Dijon, FRANCE

E-mail address: jcrisp@u-bourgogne.fr 